\title{
The development and the testing of a polyurethane (Biomer) $^{\dagger}$ arterial prosthesis
}

\author{
DAVID ANNIS \\ Institute of Medical and Dental Bioengineering, Royal Liverpool Hospital, P O Box 147, \\ Liverpool L69 3BX, UK
}

\begin{abstract}
A novel small diameter (3.8 mm ID) arterial prosthesis has been designed, manufactured and tested as a replacement for the carotid artery in the dog.

The graft is compliant in the longitudinal, circumferential and radial directions. It. is anisotropic with compliances similar to those of the natural canine carotid artery. It is flexible, the inner blood contacting surface being smooth to the naked eye. It is composed of micro-fibres of $1.0 \mu \mathrm{m}$ diameter, electrostatically drawn from a solution of polyetherurethane urea and deposited onto a rotating steel mandrel. Of 22 grafts confirmed by ultra-sound Doppler waveform analysis to be patent at 2 weeks after implantation, 17 were patent when removed at 2 years.

The histological features of the excised grafts are described.
\end{abstract}

Keywords. Artery grafts; compliant; microfibrous; polyurethane; patency; histology.

\section{Introduction}

There is a need for a synthetic arterial prosthesis having a luminal diameter between 3.0 and $6.0 \mathrm{~mm}$, for the restoration of flow through the smalker arteries of the body and, in particular, for femoro-popliteal and for aorto-coronary artery bypass, when a suitable autologous vein is not available for transposition. The mere reduction in the scale of construction of the larger woven and knitted textile grafts in common use has not met the need of small vessel replacement.

It is widely acknowledged that textile grafts, where the internal diameter is $6.0 \mathrm{~mm}$ or less, perform poorly (Herring et al 1982). The introduction into surgical practice of extended polytetrafluoroethylene (PTFE) grafts having an internal diameter of $6.0 \mathrm{~mm}$ was a small step forward. However, after ten years experience of their use it is clear that the expectation of long maintained patency afforded by their use falls far short of that afforded by the use of the patient's own saphenous vein (Walley et al 1978; Brewster et al 1983). The consequence of an increasing awareness of this limitation in the use of textile tubular devices of rigid polymer fibres has been the realization that there must be a fundamental redesign of small diameter arterial prostheses using new materials and new methods of manufacture.

The development of blood-tolerant elastomers, in particular poly(etherurethane) and poly(etherurethane urea) (Boretos 1980; Lelah et al 1983) and the means of fabricating these elastomers by a variety of techniques to form tubular structures, has made it possible for us to design an arterial prosthesis having mechanical properties much closer to those of natural arteries.

The mechanical properties of both natural arteries and some synthetic grafts have been extensively studied (Abbot and Cambria 1982; Baird et al 1977; How and Clarke 1984), there being a widely held belief based, it should be said, on very little

\footnotetext{
TTrademark
} 
documented evidence, that a mismatch of compliance between a synthetic graft and an artery to which it is attached can result in disturbances of flow that may lead to platelet adhesion and the further aggregation of platelets leading to thrombus formation. A mismatch of compliance has also been suggested as a cause of an everprogressing narrowing at the anastomosis between the synthetic graft and the natural artery, so called neo-intimal hyperplasia (Clowes et al 1985).

This report describes a small diameter arterial prosthesis of our design (Fisher et al 1985) when used as a carotid artery replacement in the dog and speculates on the reasons for the good long-term performance of the graft. The graft is a compliant, flexible microfibrous tube of wall thickness $0.35 \mathrm{~mm}$ and internal diameter $3.8 \mathrm{~mm}$. The wall of the graft is formed of fibres of Biomer polyurethane 1-2 $\mu \mathrm{m}$ in diameter with spaces of no more than $20 \mu \mathrm{m}$ between the fibres. The process of electrostatic spinning by which the graft is made allows control of the elastic modulus of the graft both in its axial and circumferential direction such that the anisotropy of the graft can be made to closely resemble that of the host carotid artery it replaces. Also, the graft, in its thickness of wall and in its luminal diameter, closely matches the dog carotid artery.

The experimental arterial prostheses were implanted into dogs as replacements of the common carotid artery. In any study of the performance of an implanted medical device the outcome is greatly influenced by the surgical technique applied to implant it and the precision with which the surgical protocol has been followed. For this reason we describe our surgical protocol in detail.

One surgeon (L De Cossart) used a rigidly standardised surgical technique to implant 26 consecutive Biomer grafts to replace $4 \mathrm{~cm}$ lengths of the carotid arteries of Beagle dogs. The grafts were $3.8 \mathrm{~cm}$ long and had an internal diameter of $3.8 \mathrm{~mm}$.

Under general anaesthesia the common carotid artery is approached through a long mid-line incision from the thyroid cartilage to the sternum and by a muscleseparating approach to the carotid sheath. The carotid artery is exposed for a length of about $12 \mathrm{~cm}$ but, before its mobilisation, the carotid sheath is infiltrated with $10 \mathrm{ml}$ of $2 \%$ lignocaine to minimise the arterial spasm which invariably occurs during mobilisation. Initially, mobilisation is limited to two, $1 \mathrm{~cm}$ lengths of artery, $12 \mathrm{~cm}$ apart. Heparin is given intravenously $(1 \mathrm{mg} / \mathrm{kg}$ body weight). Two DeBakey arterial clamps, $10 \mathrm{~cm}$ apart, and attached by an adjustable bar, are then placed on the mobilised parts of the artery, the distal clamp first. The artery is next fully mobilised between the clamps and a $4 \mathrm{~cm}$ segment of artery is excised. The adventitia of the artery is cleared from the cut edge of the artery and any clot within the lumen of the artery washed out with saline. The proximal anastomosis is performed first using 12 everting sutures of $6 / 0$ Prolene on an $8 \mathrm{~mm}$ needle, taking bites of the artery and the graft of not more than $1 \mathrm{~mm}$ width and placing the sutures approximately $1 \mathrm{~mm}$ apart. The 12 o'clock and 6 o'clock position sutures are placed and tied first. The posterior suture line is completed by turning over the artery and graft and a central suture is then placed and tied. Two further sutures are placed in each half of the back wall of the anastomosis. The artery is then turned back and the front wall anastomosis performed in a similar manner. Having completed the first anastomosis between the graft and the cut end of the artery one sees that the other cut end of the artery has shortened and retracted within the linked DeBakey clamps leaving a gap of several centimetres between it and the free end of the graft. An anastomosis could be made but only under excessive tension. 
To relieve this tension the two DeBakey artery clamps are brought closer to each other and held at a distance apart that brings the end of the cut artery and the end of the graft into close apposition. The second anastomosis can now be completed without tension by the method described for the first union. The clamps are removed and at this point it is observed that there is no bleeding from stitch holes and only a small amount of plasma appears on the outer surface of the graft. Protamine sulphate $(0.5 \mathrm{mg} / \mathrm{kg}$ body weight) is then given intravenously to reverse the effect of heparin. The flimsy carotid sheath is now closed lightly around the graft and meticulous attention given to securing complete haemastasis before the incision is closed.

All dogs receive Aspirin $75 \mathrm{mg}$ twice daily and Dipyridamole $100 \mathrm{mg}$ twice daily. This treatment is started one week before surgery and, in 18 dogs, continued throughout the course of the study. 8 dogs have their treatment gradually withdrawn during the first month after surgery.

\section{The assessment of patency of the 'Biomer' arterial prostheses and the preparation of excised specimens for histopathological examination}

The patency of the grafts was assessed by fortnightly ultrasound "duplex" scanning. The duplex scanner is a real-time B-mode imager (Toshiba SAL $50 \mathrm{~A}$ ) and a $2.4 \mathrm{MHz}$ pulsed Doppler flowmeter (Toshiba SDL-D1A). During the earlier part of the study the accuracy of the information so obtained was confirmed by the direct observation of the graft by surgical exploration. Having validated the method, we subsequently found it unnecessary to explore the grafts.

Regular ultrasound scanning was continued for two years after the implantation of the grafts, at which time the grafts together with the adjacent artery were surgically exposed under general anaesthesia and, with normal blood pressure maintained, cannulae were inserted into the carotid artery both proximal and distal to the graft. Having isolated between clamps the length of carotid artery bearing the graft, the cannulae were used to gently irrigate the specimen. Easy passage of Ringer lactate solution indicated patency of the graft.

To obtain samples for both light and electron microscopy, a gluteraldehyde, formaldehyde phosphate buffered fixative was used to irrigate the specimen, the fixative being delivered at a pressure of $120 \mathrm{~mm} \mathrm{Hg}$. Fixation for $10 \mathrm{~min}$ was sufficient to maintain the original luminal diameter of the segments of carotid artery attached to the graft. The specimen was further fixed by immersion in the same fixative.

Histopathological study was the definitive means of confirming patency and of observing the nature of the healing response of our graft.

\section{Results}

As the patency rate of grafts in the group of dogs receiving Aspirin and Dipyridamole throughout the study did not differ from the group in which the drugs were withdrawn after 4 weeks, they have been combined to form a single series of 26 consecutive graft implantations. Five grafts failed early (i.e. within two weeks of implantation) and four more failed later, between the 59th and 301st day after implantation. 
3.1 Biomer-grafts patent at 2 years after implantation-the gross and histopathological findings

Seventeen Biomer polyurethane arterial prostheses were patent two years after implantation (17/26). One was not removed but retained to study patency in excess of two years. Sixteen specimens were therefore available for gross and histopathological study.

3.1a Naked eye appearance of patent grafts at the time of removal: The specimens were observed during life at the time of removal under general anaesthesia. Approach to the graft was through natural tissue planes and the graft was easily identified both visually and by palpation. Although there were minor variations, in general the graft was clothed in a delicate, almost transparent, covering that was attached to the surface of the graft and from which it could not be separated. The suture knots were clearly visible through this covering with little more than finger dissection. The carotid arteries proximal and distal to the graft pulsated and appeared to be normal.

3.1b Naked eye appearance of all patent grafts after in-vivo fixation: After fixation, the grafts, with one to two centimetres of the adjacent carotid artery attached at each end, were cut longitudinally to form two separate gutter-like halves. Naked eye inspection of the inner blood-contacting surfaces showed them to be free from thrombus either wholly or with occasional small flattened plaques of mature thrombus. At each end an almost transparently thin 'pannus' extended across the line of anastomosis onto the inner surface of the graft. The 'pannus' extended onto the graft for approximately $1 \mathrm{~cm}$, gradually thinning, the limit of its extension usually being hardly visible as it merged with the rest of the inner surface but occasionally it was slightly thickened at its leading edge. This thickening, however, only slightly reduced the diameter of the graft, it did not seriously restrict flow. Between the limits of the inward extension of pannus, the inner surface of the graft was pale, smooth and glistening with only an occasional patch of pigment and no adherent thrombus visible. The outer surface of the graft bore a firmly adherent thin covering of connective tissue throughout its entire extent.

These appearances are noteworthy for the absence of narrowing at or near the anastomosis, and the presence only occasionally of flat patches of mature thrombus on the inner surface of the graft.

3.1c Microscopical appearances of the graft and adjacent artery: The two halves of the fixed specimen were further divided and blocks were made for histopathological study. The inner surface was also prepared for scanning electron microscopy.

The major histopathological findings were similar in all specimens. Haematoxylinand eosin-stained longitudinal sections of the anastomosis and the adjacent graft confirmed the presence, in all, of a thin 'pannus' nowhere more than $70 \mu \mathrm{m}$ thick, extending onto the inner surface of the graft but gradually thinning to end about $1 \mathrm{~cm}$ in from the anastomosis. It was composed of smooth muscle and fibrous tissue covered by a continuous monolayer of endothelium-like cells. The detailed histopathological findings will be published elsewhere. 
It is of great interest, that the inner surface of the graft specimen, between the limits of the inward extension of the pannus, was also covered by a single layer of closely adherent endothelium-like cells. These cells were attached directly to the inner fibrous surface of the 'Biomer' graft without the intervention of connective or muscle tissue. The extensive presence of these endothelium-like cells was confirmed by scanning electron microscopy. The completeness of cover has not yet been fully studied, but it can be said that cover has been complete whenever it has been sought, including the luminal surface at the centre of the graft, the area most distant from the lines of anastomosis.

A section of the wall of the graft shows it to have been penetrated by fibrous tissue both from the artery ends across the anastomoses and from the surrounding bed in which the graft lies. It is noticeable, however, that the healing connective tissue nowhere extends into the surface of the graft to a depth greater than $50 \mu \mathrm{m}$. In consequence, the bulk of the wall of the graft is free of living tissue, being filled by pink amorphous material and the occasional collection of piknotic nuclear material.

\subsection{Biomer-grafts that failed within the 2-year period of study-gross and microscopic findings}

3.2a Early failure: We have defined early failure as occlusion occurring within the first two weeks after implantation. The mode of failure in all five grafts was similar and resulted from the formation of a localised thrombus on the line of the anastomosis. Examination of the removed specimens showed that a small thrombus had formed that gradually increased in size by the accumulation of more thrombus to the point at which it was sufficiently large to occlude the lumen of the graft. When fully formed the thrombus was pear-shaped, the narrow extremity being attached at the site of one, or occasionally two, of the 12 sutures of the anastomosis. Though composed wholly of thrombus there were early indications of organisation entering the 'stalk' of the thrombus. In one example it was observed that a suture at the origin of the thrombus had been loosely tied, there being a small loop of suture material lying within the lumen. In no instance had thrombus formed on the luminal surface other than at the line of anastomosis.

3.2b Later failure: Four grafts failed later, on the 59th, 83rd, 98th and 301st day. The cause of failure varied but common to three of them was the presence of a perigraft haematoma. In one, the haematoma occurred after exploration of the graft when, early in the series, we used direct surgical observation of the graft to validate our non-invasive ultrasound method of assessment of patency. A large haematoma followed exploration. It discharged and became infected. The graft thrombosed and indeed was extruded through a discharging wound sinus. Haematoma surrounding the graft was also responsible for two other failures. In one, an organising perigraft haematoma created a thick-walled spindle-shaped fibrous capsule encasing the whole length of the graft. With the contraction of the maturing fibrous tissue the graft shortened and buckled into a sub-capsular cavity that contained old altered blood. In the other a fluid-filled cyst surrounded the graft which was associated with the separation of the fibrous inner lining of the graft to form a cylindrical tubular membrane. Its internal rupture resulted in thrombosis of the graft. 
A similar cause of failure was seen in the case of the fourth failure at 301 days after implantation, although there was no evidence of perigraft haematoma. The cause of the detachment of the inner lining membrane is uncertain, but a similar phenomenon has not been observed in any of the 17 grafts that have remained patent for two years or longer.

\section{Discussion}

The good longer-term patency of this series of $3.8 \mathrm{~mm}$ ID 'Biomer' polyurethane grafts of our design can be ascribed to the combined benefits of a number of novel features. Though microfibrous and porous, the inner surface is smooth to the naked eye and in consequence, might be expected to minimise energy loss by friction during the passage of blood. The smooth surface should also minimise turbulence during flow, which, in turn, might make thrombus formation less likely.

The wall of the graft is sufficiently porous to permit the slow entry of blood plasma on release of the artery clamps after the completion of the anastomoses. Here it coagulates to prevent the continuing passage of plasma. It is of interest to note that, on releasing the clamps a small quantity of clear plasma appears on the outer surface, the fine structure of the wall of the graft preventing the passage of red blood cells.

The Biomer poly(etherurethane urea) from which the graft is constructed is known to have excellent compatibility with blood, (Boretos et al 1975; Boretos 1980; Lelah et al 1983) especially important during the early period of residence of the graft. The growth of apparently healthy endothelium-like cells on the inner surface of the graft in direct contact with 'Biomer' fibres forming the inner surface of the graft, that is, without the intervention of fibrous tissue and muscle, is itself a sufficient testimony of the compatibility of Biomer with tissue cells and if complete, should ensure continuing prevention of thrombus formation.

The inability of new capillaries to penetrate deeply into the wall of the graft from without is another especially important feature of the graft. Capillaries cannot penetrate because the spaces between the fibres are too small to accommodate them. It has been shown that when capillaries grow through the wall of a graft they carry with them the formative elements of fibroblast and smooth muscle cells. In an elegant and important study Clowes et al (1986) have shown that when the wall of a porous Gore-Tex ${ }^{\dagger}$ (PTFE) graft has spaces large enough to allow the passage of capillaries they will rapidly reach the inner blood contacting surface, each forming a nidus from which will spread endothelium, muscle and collagenous fibrous tissue to quickly colonize the inner surface by their confluence. They have shown also that the newly formed 'pseudo-intima' continues to increase even after cover is complete. It is possible that this pseudo-intimal growth may continue to the point of occlusion of the lumen of the graft.

In the same publication they showed that a Gore-Tex graft that would not admit ingrowing capillaries developed an endothelial cover that was virtually limited to the developing 'pannus' at the ends of the graft.

Our Biomer polyurethane graft, being a barrier to all but the most superficial capillary ingrowth, is not colonized along its whole length by new intima, but only by endothelium-like cells previously described. This and the absence of fibrous and

†Trademark 
muscle tissue beneath the monolayer of endothelium-like cells we believe to be unique to our graft.

Another important consequence of the inability of healing collagenous tissue to deeply penetrate our graft is the retention by the graft of its compliance. Using realtime ultrasound scanning we have shown that even after two years residence as an arterial replacement our graft remains pulsatile, retaining, to a degree, the circumferential compliance it had at the time of implantation. We designed the compliance of the graft to be similar to that of the natural carotid artery in the belief that as we have earlier stated, there are good theoretical reasons why a mismatch of compliance at an anastomosis between a synthetic graft and a natural artery may cause disturbances of flow and the creation of points of focal stress near an anastomosis sufficient to cause thrombus to form. It has also been held responsible for the later development of progressive narrowing at an anastomosis, due to pseudo intimal hyperplasia, leading to occlusion.

Apart from these theoretical explanations for the good performance of our graft it should be remembered that good performance of an arterial prosthesis is highly dependent upon a good surgical technique of implantation, and that a graft, in its "handling", should assist the surgeon in application of good surgical technique.

Our graft cuts cleanly without fraying and a 6/0 'Prolene' $\dagger$ suture can be placed within $1.0 \mathrm{~mm}$ of the cut edge of the graft and all necessary tension applied to it without its cutting out. The graft being of a fibrous elastic material, the needle holes close tightly around the suture preventing needle-hole bleeding.

Whatever the theoretical benefit of circumferential compliance may be, its practical advantage is the ease with which it is possible for the surgeon to evert the cut end of the graft sufficiently to match the eversion that is possible with the natural artery. It seems important to us that the technique of suturing should evert both the natural artery and the graft in such a way as to minimise, if not wholly prevent, the in-turning of any part of the exposed arterial wall towards the lumen at the site of anastomosis. We have shown that early failure with our graft was due to thrombus forming on the anastomotic line. This has ceased to be a problem as our surgical technique improved and our experience increased.

We conclude therefore that, because the mechanical properties of the graft so closely resemble those of the natural vessel to which it is attached, we have been able to incorporate into the design of our graft a number of structural and mechanical features that, on theoretical grounds, should be advantageous. We have also made it possible for the surgeon to apply to the union of a synthetic artery to a natural artery those well-established techniques of anastomosis that have evolved over the years for the union of natural vessels.

A clinical trial, in man, of the use of an arterial prosthesis of similar construction but of larger dimensions $(6 \mathrm{~mm}$ internal diameter and approximately $25 \mathrm{~cm}$ long) has demonstrated its usefulness and its satisfactory performance where its use has been limited to the creation of an arteriovenous communication to shunt rapidly moving arterial blood at arterial blood pressure into subcutaneous veins. This is required for the care of patients in the late stage of kidney failure when life depends upon the regular connection of the patient to an extra corporeal dialysing machine. The use of the arterial prosthesis for the wider purpose of the replacement or bypassing of obstructed arteries in the peripheral arterial circulation and for

\footnotetext{
†Trademark
} 
coronary artery bypass must await further evidence of the long-term durability of the device after long residence in the body.

\section{References}

Abbot W M and Cambria R P 1982 in Biologic and synthetic vascular prostheses (ed.) J C Stanley (New York: Grune and Stratton) p. 189

Baird R N, Kidson I G, L'Italien G J and Abbot W M 1977 Am. J. Physiol. 223 H568

Boretos J W 1980 Pure Appl. Chem. 521851

Boretos J W, Pierce W S, Baier R E, Leroy A F and Donachy H J 1975 J. Biomed. Mater. Res. 9327

Brewster D C, LaSalle A J, Robinson J G, Strayhorn E C and Darling R C 1983 Surg. Gynecol. Obstet. 157437

Slowes A W, Gown A M, Hanson S R and Reidy M A 1985 Am. J. Pathol. 11843

Jlowes A W, Kirkman T R and Reidy M A 1986 Am. J. Pathol. 128220

Fisher A C, How T V, de Cossart L and Annis D 1985 Trans., Am. Soc. Artif. Intern. Organs 31324

Herring M B, Dilley R, Peterson G, Wiggans J, Gardner A and Glover J 1982 J. Surg. Res. 32138

How T V and Clarke R M 1984 J. Biomech. 17597

Lelah M D, Lambrecht L K, Young B R and Cooper S L 1983 J. Biomed. Mater. Res. 171

Walley D. James P M. Meredith J H, Todd S K and Ogburn N 1978 Arch. Surg. (Chicago) 113863 